
3
Research Square
Preprints are preliminary reports that have not undergone peer review.
They should not be considered conclusive, used to inform clinical practice, or referenced by the media as validated information.

\title{
The Efficacy of Computer-Assisted Cognitive Behavioral Therapy on Psychobiological Responses in Patients Undergoing Functional Endoscopic Sinus Surgery: A Randomized-controlled Clinical Trial
}

Yang Yang ( $\nabla$ yangyang_880914@163.com )

First Hospital of Shanxi Medical University

Yuling Li

First Hospital of Shanxi Medical University

Haibin Zhang

First Hospital of Shanxi Medical University

Yong Xu

First Hospital of Shanxi Medical University

Binquan Wang

First Hospital of Shanxi Medical University

\section{Research}

Keywords: computer-assisted cognitive behavioral therapy, psychobiological response, functional endoscopic sinus surgery, anxiety, insomnia, cortisol

Posted Date: October 14th, 2020

DOl: https://doi.org/10.21203/rs.3.rs-90810/v1

License: (c) (i) This work is licensed under a Creative Commons Attribution 4.0 International License. Read Full License

Version of Record: A version of this preprint was published at Perioperative Medicine on August 19th, 2021. See the published version at https://doi.org/10.1186/s13741-021-00195-3. 


\section{Abstract}

\section{Background}

Previous reports have shown computer-assisted cognitive behavioral therapy (CCBT) could improve mood and sleep for chronic health conditions and some anesthetics can help reduce preoperative anxiety, stabilize hemodynamics and lessen hormone response. But it is unclear whether CCBT could help adults with short-lived acute stressor (e.g. surgery) to experience similar responses.

Objective

This study aimed to assess the effects of CCBT on surgery-related physiological and psychological indices (mood, insomnia, vital signs and stress hormone) in patients undergoing functional endoscopic sinus surgery (FESS) during the perioperative period.

Methods

Participants were randomly assigned to a usual care (UC: disease care and psychological care) group ( $\mathrm{n}$ $=50$ ) or a computer-assisted cognition behavior intervention (CCBT: cognitive therapy and behavioral relaxation) group ( $n=50)$. The primary outcome was state anxiety measured at baseline (T1) using the State Anxiety Inventory (SAI), with repeat assessment conducted 1 hour before anesthesia administration (T2) and post-intervention (T4). Secondary outcomes, including symptoms of depression and insomnia, were recorded at T1, 48 hours after surgery (T3), and T4. The stress hormone were assayed at T1 and T2, and participant satisfaction with intervention was assessed at T4. Blood pressure, and heart rate were collected at above four time points. Repeated-measure ANOVA models were then used to compare results between-two groups and analyze changes in anxiety, depression, and insomnia levels among participants.

Results

At T2/T3 and T4, compared to the UC group, the CCBT group showed reduced anxiety $(F=17.10, p<.01$ and $F=38.54, p<.01)$, and relieved insomnia symptoms $(F=10.10, P=.002$ and $F=9.46, P=.003)$. Furthermore, the secondary analysis revealed a significant reducer in the participants falling above the clinical cut-off scores in the CCBT group as compared with UC group in SAI scores at T2 (72\% vs $88 \%, \mathrm{p}=$ $.04)$ and in AIS scores at T4 ( $4 \%$ vs $22 \%, p=.01)$. For stress hormone, not only the increase in cortisol levels in the UC group was significantly higher at T2 compared to T1 ( $p=.001)$; but also cortisol levels for UC group compared to the CCBT group was found to have a significant effect ( $p=.024)$ at T2. For vital signs, the change of BP and HR were different significantly between-two group at T2 $(p<.01)$. Moreover, the CCBT group showed higher satisfaction scores with Psychological care, Psychosomatic management and total score than the UC group.

Conclusion 
The CCBT appeared to reduce anxiety level and insomnia symptom, stabilize preoperative hemodynamics and cortisol level in patients undergoing FESS under general anesthesia. Participants also evaluated the CCBT positively, suggesting that it may represent an effective adjunct in preparing patients for surgery.

Trial state

The study was registered with the Chinese Clinical Trial Registry (ChiCTR1900025994) on September 17, 2019. http://www.chictr.org.cn/listbycreater.aspx/ChiCTR1900025994 The study protocol was conducted with the consent of the Ethics Committee of First Hospital of Shanxi Medical University in China on July 31, 2019 (document number [2019]K-SK028)

\section{Introduction}

Chronic rhinosinusitis (CRS) is a chronic local inflammatory disease with a relatively high incidence. The overall prevalence of CRS was $8.0 \%$ in seven centers in China [1]. Functional endoscopic sinus surgery (FESS) is a new technology for nasal surgery and become the mainstream technology in the treatment of sinusitis. FESS represents the first choice for surgical treatment once CRS becomes refractory and is no longer responsive to medication [2]. Surgery as stressor can affect physical and psychological response through autonomic and neuroendocrine [3]. Many studies have found that preoperative anxiety is common among adult patients scheduled for elective procedures and general anesthesia, and the incidence is about $11 \%$ to $80 \%[4,5]$. The preparation in the preoperative waiting room (T2) is the trigger point for anxiety, and more than $80 \%$ of adult patients have a high level of anxiety [6]. Surgical patients frequently report postoperative sleep disturbances, mainly occurring in patients under general anesthesia [7]. The incidence of postoperative sleep disorder is even more than $80 \%$ [8]. Yang et al. found that more inferior sleep quality was correlated with anxiety and depression [9]. Song et al. thought that general anesthesia affected sleep rhythms, which contributed to postoperative sleep disorders by changing melatonin levels and/or circadian clock genes in vivo [10]

Stress not only would result in too much cortisol, reduced heart rate variability, heightened BP, or impaired immunity, but also alter the production of stress mediators in ways that can both increase and blunt, both upregulate and downregulate [3]. Patients with FESS experience psychological and physical stress during the process of diagnosis and treatment. In such stressful situations, hyperactivity of the hypothalamicpituitary-adrenal (HPA) axis is known to be associated with the onset of anxious and depressive symptoms [11]. The secretion of corticotropin-releasing hormon ( $\mathrm{CRH})$ acts on the pituitary gland to induce the secretion of adrenocorticotropic hormone (ACTH), which in turn acts on the adrenal gland to stimulate the production and release of cortisol $[11,12]$. The preoperative waiting (T2) as a trigger point for high-level anxiety may be lead stress markers to increase and serum cortisol level are altered in response to anxiety. Nasim ea al in their study recommended that measure patients' serum cortisol levels at their admission and compare the values with preoperative levels to investigate the effectiveness of training before surgery [13]. 
Regulating stress response reasonably should be concerned for surgeons and anesthesiologists [14]. To this aim, our present study aimed to develop a cheap, simple, and standardized approach for identifying and relieving stress response in patients during the perioperative period.

Cognitive-behavioral therapy (CBT) is the first choice recommended by the FDA for anxiety disorders and can ameliorate negative emotions and bodily responses by correcting maladaptive thoughts [15]. Dao et al. found that CBT was both feasible and acceptable to improve symptoms of depression or anxiety in patients undergoing coronary artery bypass graft surgery. Additionally, CBT has shown to improve healthrelated quality of life (HRQOL) and reduce the in-hospital length of stay [16]. Although CBT is practical and suitable for patients undergoing surgery, there are barriers to the implementation of psychotherapy, such as time and travel burdens for patients to attend face-to-face sessions, a shortage of adequately trained therapists, high costs, the potential stigma associated with seeking professional help, and a lack of accessibility in remote areas $[17,18]$. Computer-assisted cognitive behavioral therapy (CCBT) has been suggested as an alternative method to address these limitations.

CCBT is a remote mental-health intervention model based on computer technology and CBT via a computer interface or smartphone application. CCBT has developed as an adjunct method for patients receiving effective psychotherapy in both psychiatric and medical settings [19]. Not only has CCBT been shown to be effective in psychiatric disorders [20], but also shown to be useful as an adjunct treatment in mental illness associated with somatic diseases, such as multiple sclerosis [21], irritable bowel syndrome [22], sickle cell disease [23], depression after stroke [24], and dental anxiety [25]. However, no previous studies have used a CCBT software package to ameliorate negative emotions and insomnia associated with surgery. In the present study, a new CCBT program named "computer-assisted psychosomatic cognitive behavioral therapy during perioperative period (CPCBT-Perio)" was developed and optimized based on Enhanced Recovery After Surgery (ERAS) and CBT theory. Unlike past CCBT, the content of the optimized CPCBT-Perio 1.0 version includes surgical nursing knowledge, anesthesia video instruction, postoperative recovery, and psychotherapy technical guidance.

Accordingly, the initial aim of the current study was to develop a computer-assisted psychosomatic CBT software package (CPCBT-Perio), including five sections procedure based on CBT theory for Chinese patients with FESS during the perioperative period. The second aim was to conduct a pilot RCT of CPCBTPerio. The pilot aimed to test the efficacy of CPCBT-Perio procedures and explore the efficacy of decreasing symptoms of anxiety, depression, and insomnia, and the effect on BP, HR, stress markers and satisfaction compared to an attention-matched control condition. The hypotheses of the study are as follows:

1. CCBT group will show lower anxiety level and concentration of cortisol and adrenocorticotropic hormone (ACTH) than those in the UC group at preoperative waiting room.

2. CCBT group will show far less depression and insomnia symptom than those in the UC group postoperation. 
3. CCBT group will show more stabilize hemodynamics (BP and HR) than those in the UC group during perioperative period.

4. CCBT group will express higher satisfaction compared to the UC group before discharge.

\section{Materials And Methods}

\subsection{Study design}

The present study was a prospective, randomized, controlled, and single-blind trial with one experimental arm (CCBT group) and one control arm (UC group). We independently randomized participants to receive either five sessions of CCBT + UC or UC alone. The primary study outcomes were state anxiety compared between two groups. Secondary outcomes included symptom of depression and insomnia, BP, HR stress biomarkers, and satisfaction with intervention compared between two groups. Participants were recruited from the Otolaryngology Department through the First Hospital of Shanxi Medical University from September 20, 2019, to January 10, 2020. All interested and eligible participants completed full informed consent prior to study procedures. Designing and reporting of this clinical trial reference CONSORT guidelines [26] (see Supplementary Table 1 for CONSORT Checklist).

\subsection{Participants}

Inclusion criteria were the following: (1) aged 18-60 years; (2) diagnosis of CRS and scheduled to undergo FESS within a week; (3) general anesthesia can be acceptable as a form of anesthesia; (4) be able to answer the questionnaires and participate in the interventions; (5) be willing to participate in the study. Exclusion criteria included the following: (1) diagnosis of definite history of psychiatric illness, substance abuse/dependence within 12 months before enrollment; (2) undergoing a surgery that was postponed for more than a week or changed to emergent surgery; (3) currently receiving any psychiatric or psychological treatment, including psychiatric medication; (4) the Patient Health Questionnaire Depression Scale-9 (PHQ-9) score $\geq 20$ and (5) those who had serious cardiovascular and respiratory diseases.

\subsection{Study assessments}

A psychological therapist holding M.A.degree had given training to researchers aiming at the consistency of psychological assessment. The intervention designer is a doctoral student in nursing who had provided intervention flow and some observing details to researchers. A team of researchers (two nursing graduate students and a psychological therapist) collaborates with hospital staff from the Otolaryngology Department enrolled patients. Participants were identified and confirmed from surgicalplan lists by the Electronic Medical Record System. Then they were approached by researchers and written informed consent would be obtained according to the ethical requirements before any study procedure. The next step was that the severity of emotional distress(depression) was assessed according to PHQ-9. If the patients were demonstrated severe depression or a high risk of suicide (PHQ-9 score $\geq 20$ ), they would be excluded and referred to a psychiatrist [27]. Finally, participants who had serious 
cardiovascular and respiratory diseases were excluded further. After enrollment, baseline questionnaires about demographic and medical information was collected. Participants were randomly assigned via a concealed random digital table and admission order to ensure blinding of study staff and participants until baseline assessments were completed. A study staff was along blinded to collect and analyze data for group assignment during the study.

Baseline characteristics included sex, age, education, principal diagnosis, surgery type, and anesthesia grading. The baseline measures were collected on the day after hospitalization. The measures and outcomes were obtained from patients at baseline (T1), one hour before anesthesia administration (T2), on the 48 hours after surgery (T3) and post-intervention (T4). There was no evidence of harm or unintended effects in either the intervention or control groups. (see Figure 1 that shows the schedule of study assessment, and intervention time points.)

\subsection{Study interventions}

\subsubsection{Introduction}

The potential participants were given a brief introduction about the study program before beginning the five-session intervention, especially the importance of mental health for postoperative recovery. Eligible inpatients who were willing to participate were registered and kept in touch by study staff.

\subsubsection{CPCBT-Perio software program intervention}

The CCBT group received the optimized CCBT (CPCBT-Perio) intervention in addition to UC. The CPCBTPerio is a non-internet-based computer program that includes registration and therapy conducted in a medical setting. The program is based on condition-surgery and CBT elements, which comprised five sessions, and each session took about 20 minutes to complete. The preoperative preparation time is usually only $3( \pm 1)$ days for patients with FESS since admission and postoperative recovery time is about 7 ( \pm 2$)$ days. The time points of intervention are 2-day, 1-day before operation and 2-day, 3-day, and 4-day post operation. All sessions would finish before discharge. Since this program was standardized and straightforward, a nursing graduate student assisted participants with CCBT treatment. Participants in the CCBT group entered their information into the program and were provided instructions on how to use the software, and the first treatment was administered soon after registration. Each session starts by logging into the admission number. Next time logging in, individualized treatment will continue. The treatment modules within the program included cognitive therapy, cognitive consolidation, and behavioral relaxation therapy. The primary components of the cognitive therapy module included the following:

Session 1: Preoperative psychological preparation;

Session 2: Preoperative physical preparation and introduction to the surgical environment;

Session 3: Management of postoperative pain, insomnia, and anti-thrombus; 
Session 4: Postoperative exercise and diet;

Session 5: Education on steps following hospital discharge.

The behavioral therapy module included relaxing training, such as imaginative relaxing exercise, progressive muscle relaxation, breathing exercises, relaxing sleep exercise, and a mindfulness meditation body scan. Homework as cognitive consolidation was then provided for participants to answer questions in the form of a game, in which the questions served as a review of the previous cognitive therapy module. The cognitive therapy module and behavioral therapy module were presented via video. (see Supplemental table 2 for outlines precisely the content of the sessions)

The studies have revealed that $\mathrm{CBT}$ can relieve anxiety and depression for patients with surgery [16]. The CPCBT-Perio procedure was developed based on CBT theory and method, similar to the standard CCBT. That is changing unreasonable cognition and thoughts and providing better management of stress and coping with tension. The unique feature of the CPCBT-Perio is that learning identifies core beliefs and negative thoughts about surgery and helps patients reorganize and rationalize cognition by video. Simultaneously, some skills for relaxing had been provided with coping with preoperative anxiety and postoperative insomnia. On the computer interface, they express CBT utilizing clear operational steps, highly structured multiple media interactions. The psychological knowledge about emotion management was included in the CPCBT-Perio program the same as standard CCBT. The contents of operation and anesthesia which were reviewed and passed by clinical nursing specialists and psychotherapists were unique and customized for surgical patients.

\subsubsection{Usual Care intervention}

In the case of UC, patients were administered the FESS routine care conducted according to the Perioperative Care Manual and Consensus on ERAS [28].To match the CCBT group, participants randomized to this condition, including five sessions, focused on education about the illness, surgery, anesthesia, and postoperative nursing. These sessions were developed in consultation with clinical nursing specialists. A 20-minute verbal briefing per session was administered to participants by study staff. Topics included the introduction of illness and surgery; Preoperative psychological and physical preparation; Postoperative disease care; Activities and diet after surgery; Education on steps following hospital discharge.

\subsection{Measures and Outcomes}

\subsubsection{Primary outcome measure}

State-Trait Anxiety Inventory (STAI) was developed by Spielberger C.D and Gorsuch R.L to measure transient and enduring levels of anxiety [29]. Historically, the STAI is considered to assess and differentiate clinical anxiety levels as the gold standard test [30]. The first part of the STAI -"State scale"(STAI-S) assesses current state of anxiety at a particular moment asking how respondents feel "right now" [29]. If detecting change over a relatively short period of time, many have opted to solely use 
the STAI-S subscale for the detection of longitudinal change. The second part of the STAI- "The Trait scale" (STAI-T) evaluates relatively stable aspects of "anxiety proneness" including general states of calmness,confidence, and security [31]. While overall reliability analysis using Cronbach's alpha shows that these scales are reliable (alpha $=0.90$ and 0.81 for State and Trait scales, respectively) [32]; we obtained a reliability value of 0.80 and 0.85 The patients remained constant visible anxiety from the afternoon before surgery to the immediate preoperative period [33] and preoperative waiting is the trigger point for high-level anxiety [6]. Anxiety and tension should be controlled or decreased before surgery and anesthesia administration (T2) to less bleeding and the smooth operation of FESS for a surgeon [14]. We administered the STAI-S assessment at T1, T2, and T4. Past studies display that STAI-S score $\geq 38$ was considered to be moderate and severe anxiety [34].

\subsubsection{Secondary outcome measure}

The Patient Health Questionnaire Depression Scale-9 (PHQ-9) can establish provisional depressive disorder diagnoses as well as grade depressive symptom severity [27]. The Chinese version of the PHQ-9 Cronbach's alpha values of PHQ-9 was 0.83 for inpatients in general hospitals study [35], while we obtained a value of 0.80 . The physical discomfort such as nasal tamponade, pain, bleeding and postoperative sleep disturbances may be come along after surgery [36]. Depression symptoms may gradually appear with the complications after surgery. Athens Insomnia Scale (AIS) was developed by Soldatos to quantify sleep difficulty based on ICD-10 criteria [37]. As assessed by Cronbach's alpha, internal reliability was 0.89 in the original study [37], while we obtained a value of 0.90 . General anesthesia and physical discomfort can cause postoperative insomnia [38]. Considering the physical discomfort of the patients after surgery, we chose to assess at 48 hours after surgery. We administered the PHQ-9 and AIS assessment at T1, T3, and T4. The PHQ-9 score $\geq 5$ was considered a clinical depressive state [27] and the AIS score $\geq 6$ was a cutoff distinguishing between insomnia patients and controls in $90 \%$ of cases [39]

The psychological state before and after operation often affects the stability of vital signs of patients [40]. The values of Blood Pressure (BP) including of Systolic Blood Pressure (SBP) and Diastolic Blood Pressure (DBP) and Heart Rate (HR) are collected at T1 T4.

Abelson et al. found that cognitive intervention can significantly reduce the concentration of cortisol and ACTH in patients by adjusting the function of the hypothalamic-pituitary-adrenal (HPA) axis [41]. Peripheral venous blood was collected at baseline and before anesthesia, the latter of which is associated with increased anxiety [42]. We collected venous blood of the patients who were under inpatient between 6:00 a.m. and 8:00 a.m. on the day of admission (T1) and operation (T2). Blood samples were taken before breakfast to minimize the effects of circadian variation. A single venipuncture collected $5 \sim 8 \mathrm{ml}$ blood into purple and yellow plain tubes (with coagulants and separation gel). After standing at room temperature for $30 \mathrm{~min}$, the blood sample centrifuged for $15 \mathrm{~min}$ at 1,800 g. Plasma Cor and ACTH levels were measured by chemiluminescence assay (CLIA) and microparticles CLIA. These 
measurements aimed to determine whether CCBT can result in a change of stress biomarkers, such as Cortisol and ACTH.

We also collected data on participant satisfaction, in which participants completed a brief 10-item satisfaction survey on either the day of discharge (T4) or the day of withdrawing from the study if applicable. Internal reliability for this scale was high at 0.87 (Cronbach's alpha) (see Supplemental Information table 3 for Satisfaction Survey Questionnaire).

\subsection{Data Analysis}

According to a previous study [43], the average value of SAI in control subjects is $44.7 \pm 10.3$. To have a difference greater than 5 in average levels of state anxiety between groups, and with a power of 0.80 at the $5 \%$ significance level, we estimated a sample size of 46 patients per arm according to the value of the standard deviation, $\sigma=10$ (PASS 11.0). Allowing for a $10 \%$ dropout rate, approximately 50 cases were ultimately included per group.

Analyses of the outcomes were conducted by IBM SPSS Statistics, version 23.0 (2010 SPSS Inc., IBM Company, Armonk, NY, USA). Descriptive statistics (percentages, means, and standard deviations [SD]) were used to summarize baseline participant characteristics and scores on the self-report measures. Chisquare and Student $t$ tests were performed to test differences of between-group on baseline characteristics and changes from baseline in the outcome measures. We used linear mixed models in SPSS to analyze between-group differences on each outcome at each study time point. Anxiety state was the primary outcome with the T2 (i.e., before - anesthesia) timepoint representing the main study time point, and the T4 (post - intervention) time point as an exploratory outcome. To evaluate the impact of the intervention conditions on changes in the primary outcome (STAI-S), as well as secondary outcomes (PHQ-9 and AIS), 2 (intervention) × 3 (time) repeated-measures ANOVA models were conducted. Effect sizes were also calculated for each outcome variable by dividing the mean difference between time points by the pooled standard deviation [44]. Two-way ANOVA compared Cortisol and ACTH between two groups. Mann-whitney $U$ tests were used analyzed the data of nonnormally distribution variables. All of the tests were two-sided, and statistical significance was set at $p<0.05$.

\section{Results}

\subsection{Sample Characteristics}

120 patients were interested in participation and assessed eligibility. Following inclusion and exclusion criteria, finally 100 patients were approached for the study including declining to participate $(n=10)$, PHQ9 score $\geq 20(n=2)$, serious cardiovascular and respiratory diseases $(n=7)$, canceling surgery $(n=1)$ Fifty participants were randomly assigned to the CCBT group, and fifty participants were randomly assigned to the UC group. Figure 2 shows the CONSORT flow diagram, including information about study exclusion and the sample size for analysis. Table 1 summarizes the demographic data of the two groups and demonstrates that they are well-matched in terms of baseline patient characteristics. 
Table1 Characteristics of the study population

\begin{tabular}{|c|c|c|c|c|}
\hline Variable & UC group $(N=50)$ & CCBT group $(\mathrm{N}=50)$ & tor $\chi^{2}$ & $\mathrm{p}$ \\
\hline Age $($ Mean $\pm S D)$ & $43.08 \pm 12.51$ & $42.10 \pm 12.89$ & 0.15 & 0.70 \\
\hline \multicolumn{5}{|l|}{ Gender(\%) } \\
\hline Men & $25(50.00)$ & $22(44.00)$ & \multirow[t]{2}{*}{0.36} & \multirow[t]{2}{*}{0.55} \\
\hline Women & $25(50.00)$ & $28(56.00)$ & & \\
\hline \multicolumn{5}{|l|}{ Education(\%) } \\
\hline High school graduate & $36(72.00)$ & $37(74.00)$ & \multirow[t]{2}{*}{0.05} & \multirow[t]{2}{*}{0.82} \\
\hline Nongraduates & $14(28.00)$ & $13(26.00)$ & & \\
\hline STAI-S (Mean \pm SD) & $32.02 \pm 5.89$ & $33.12 \pm 6.80$ & -1.60 & 0.39 \\
\hline STAI-T (Mean \pm SD & $34.50 \pm 8.01$ & $35.18 \pm 9.51$ & 0.17 & 0.87 \\
\hline PHQ-9(Mean \pm SD) & $3.40 \pm 3.26$ & $3.14 \pm 2.48$ & 0.34 & 0.66 \\
\hline AIS & $3.16 \pm 2.44$ & $3.60 \pm 2.16$ & -1.08 & 0.34 \\
\hline Cortisol & $307.07 \pm 111.92$ & $295.43 \pm 125.66$ & 0.40 & 0.26 \\
\hline ACTH & $37.39 \pm 24.70$ & $38.17 \pm 17.40$ & -0.14 & 0.88 \\
\hline \multicolumn{5}{|l|}{ Diagnosis(\%) } \\
\hline CRS with NP & $26(52.00)$ & $30(60.00)$ & \multirow[t]{3}{*}{1.111} & \multirow[t]{3}{*}{0.574} \\
\hline CRS(only) & $20(40.00)$ & $15(30.00)$ & & \\
\hline Other & $4(8.00)$ & $5(10.00)$ & & \\
\hline
\end{tabular}

All values are mean \pm SD or number (proportion). STAI-S, Spielberger State Anxiety Inventory. STAI-T, Spielberger Trait Anxiety Inventory. PHQ-9, Patient Health Questionnaire Depression Scale-9 item. AIS, Athens Insomnia Scale. ACTH, Adrenocorticotropic Hormone. CRS with NP, Chronic Rhinosinusitis with Nasal Polyps. CRS, Chronic Rhinosinusitis.

3.2 Comparative analysis between two groups on the STAI-S, PHQ-9, and AIS scores(see table 2 and figure 3 )

The scores of SAI and AIS in CCBT group were lower than UC group except scores of PHQ-9 at T2/T3 and T4. The effect on scores of the scales over time in the two groups were statistically significant. 
The repeated-measure ANOVA results found that $P>0.05$ conforms to the spherical hypothesis in Mauchly's Test for STAI-S, PHQ-9 and AIS. For STAI-S scores, the impact of group*time interaction was statistically significant $(F=11.342, p<0.01)$. At $T 2$, the scores in CCBT group was lower 5.04 than UC group $(F=17.10 冈 p<0.01,95 \%$ Confidence Interval $[C I]:-7.04,-2.63, E S=0.15)$. At $T 4$, the scores in CCBT group was lower 5.64 than UC group ( $F=38.54, p<0.01,95 \%$ Cl: $-7.44,-3.84$, ES $=0.28$ ). For PHQ-9 scores, the impact of treatment*time interaction was not statistically significant $(F=2.94, P=0.06)$. The effect of treatment grouping on time was statistically significant $(F=19.33, P<0.01)$. But at T3 and T4, the scores between two groups were no statistically different $(F=0.14, p=0.71$ and $F=0.08, p=0.78)$. For AIS scores, the impact of treatment*time interaction was statistically significan $t(F=8.11 \otimes P<0.01)$. At T3囚the scores in CCBT group was lower 1.26 than UC group ( $F=10.10, P=0.002,95 \% \mathrm{Cl}:-2.05,-0.47$, ES $=0.09)$. At T4, the scores in CCBT group was lower 1.26 than UC group $(F=9.46, P=0.003,95 \% \mathrm{Cl}$ : $-2.07,-0.45, E S=0.09$ )

Table 2 Between-group differences in change from baseline on study outcome measures (Mean \pm SD)

\begin{tabular}{|lllllll|}
\hline Measure & & CCBT group $₫ n=50 \rrbracket$ & UC group $(n=50)$ & $F$ & $p$ & ES \\
\hline T1 to T4 & & & & 88.64 & 0.00 & 0.48 \\
\hline STAl-S & T2 & $39.76 \pm 4.95$ & $44.80 \pm 7.06$ & 17.10 & 0.00 & 0.15 \\
\cline { 2 - 7 } & T4 & $23.34 \pm 3.37$ & $28.98 \pm 5.47$ & 38.54 & 0.00 & 0.28 \\
\hline T1 to T4 & & & & 19.33 & 0.00 & 0.17 \\
\hline PHQ-9 & T3 & $4.18 \pm 2.50$ & $3.98 \pm 2.91$ & 0.14 & 0.71 & 0.00 \\
\cline { 2 - 7 } & T4 & $1.98 \pm 1.73$ & $2.08 \pm 1.90$ & 0.08 & 0.78 & 0.00 \\
\hline T1 to T4 & & & & 11.68 & 0.001 & 0.11 \\
\hline AlS & T3 & $4.90 \pm 1.73$ & $6.16 \pm 2.21$ & 2.30 & 0.002 & 0.09 \\
\cline { 2 - 7 } & T4 & $1.92 \pm 1.86$ & $3.18 \pm 2.22$ & 9.46 & 0.003 & 0.09 \\
\hline
\end{tabular}

Results of between-group differences in change from baseline to T4 were from repeated-measures ANOVA models. STAI-S, Spielberger State Anxiety Inventory; PHQ-9, Patient Health Questionnaire Depression Scale-9 item; AIS, Athens Insomnia Scale. T1:at baseline; T2: on 1 hour before anesthesia; T3: on 48h after surgery; T4: post-intervention

3.3 The comparison about the percentage of participants above the clinical cut-off scores (see figure 4)

At T1, the percentage of participants above the clinical cut-off scores (STAl-S score $\geq 38$ ) was $30 \%$ vs $26 \%$ for the two groups $\left(c^{2}=0.19, p=0.66\right)$. At T2, the percentage was $72 \%$ vs $88 \%\left(c^{2}=4.00, p=0.04\right)$ and the percentage was 0 vs $6 \%\left(c^{2}=3.09, p=0.07\right)$ at T3. For PHQ-9, at $T 1$, the percentage of participants above the clinical cut-off scores (PHQ-9 score $\geq 5$ ) was $22 \%$ vs $26 \%$ for the two groups ( ${ }^{2}=$ 
$0.22, p=0.64)$. At $\mathrm{T} 2$, the percentage was $40 \%$ vs $36 \%\left(c^{2}=0.17, p=0.68\right)$ and the percentage was $10 \%$ vs $10 \%$ at T3. For AIS, at T1, the percentage of participants above the clinical cut-off scores (AIS score $\geq 6$ ) was $26 \%$ vs $22 \%$ for the two groups $\left(c^{2}=0.22, p=0.64\right)$. At T3, the percentage was $36 \%$ vs $54 \%$ ( $c^{2}=$ $3.27, p=0.07)$ and the percentage was $4 \%$ vs $22 \%$ at $\mathrm{T} 4\left(c^{2}=7.16, p=0.01\right)$. The results illustrated that the difference for percentage above the clinical cut-off scores (compared with zero) in STAI-S scores at T2 and in AIS scores at T4 was statistically significant between the two groups. While, the results did not show the percentage difference in PHQ-9 between two groups.

3.4 Cortisol and ACTH Levels compare of two groups at T1 and T2

The tests of between-subjects effects showed interaction of group*time was significant for cortisol level ( $F=5.84, p=0.017$ ) and not for ACTH level $(F=0.001, p=0.974)$ (see Table 3). A further test of simple effect analysis showed increases in cortisol level of the UC group were significantly greater at T2 compared to $\mathrm{T} 1[\mathrm{~F}(1,116)=11.12, \mathrm{p}=0.001]$; Increases in cortisol level at T2 were significantly UC group compared to CCBT group $[F(1,116)=5.25, p=0.024]$.

\section{Table 3 Cortisol and ACTH comparative analysis between two groups $\square$ Mean \pm SD $\square$}

\begin{tabular}{|lllll|}
\hline Measure & \multicolumn{2}{l}{ CCBT $(\mathrm{n}=30)$} & UC $(\mathrm{n}=30)$ \\
\cline { 2 - 5 } & $\mathrm{T} 1$ & $\mathrm{~T} 2$ & $\mathrm{~T} 1$ & $\mathrm{~T}$ \\
\hline Cortisol $(\mathrm{nmol} / \mathrm{L})$ & $307.07 \pm 111.92$ & $306.22 \pm 74.86$ & $295.43 \pm 125.66$ & $349.57 \pm 83.39 \Delta^{*}$ \\
\hline ACTH $(\mathrm{pg} / \mathrm{mL})$ & $37.39 \pm 24.70$ & $39.55 \pm 20.30$ & $38.17 \pm 17.40$ & $40.57 \pm 13.41$ \\
\hline
\end{tabular}

Two-way ANOVA was used to analyze data. * shows $p<0.05$, CCBT group vs UC group at the same time point. $\Delta$ shows $p<0.05$, T2 vs T1 in the two groups.

3.5 The change trend comparison about SBP, DBP and HR

The change trend comparison about SBP, DBP and HR between two group found that the trend for CCBT group were more stabilized than UC group. More important, at T2, the SBP mean value of CCBT group was lower than UC group $(127.24 \pm 16.27$ vs $146.06 \pm 20.59)(F=25.71, p<0.01)$, the same to the DBP mean value $(78.48 \pm 11.42$ vs $87.86 \pm 13.31)(F=14.30, p<0.01)$, and the HR mean value $(79.90 \pm 10.73$ vs $90.42 \pm 12.83)(F=19.78, p<0.01)$. (see Figure 5$)$

\subsection{Satisfaction survey comparisons between two groups}

Participant satisfaction was significantly higher in the CCBT group than in the UC group for all of the assessed categories except disease care $(Z=-1.58, p=.115)$ before discharge care item (Table 4$)$.

\section{Table 4 Satisfaction comparison between two groups before discharge}




\begin{tabular}{|lllll|}
\hline Item & UC group $(n=50)$ & CCBT group $(n=50)$ & Z value & $p$ \\
\hline Psychological care & $8.30 \pm 1.30$ & $9.40 \pm 0.93$ & -4.41 & 0.00 \\
\hline Disease care & $18.50 \pm 1.79$ & $18.90 \pm 1.90$ & -1.58 & 0.12 \\
\hline Psychosomatic management & $15.68 \pm 2.33$ & $18.20 \pm 2.34$ & -4.99 & 0.00 \\
\hline Total & $46.70 \pm 4.41$ & $42.46 \pm 4.58$ & -4.60 & 0.00 \\
\hline
\end{tabular}

\section{Discussion}

In the present study, we aimed to explore the efficacy of an optimized five-sessions CPCBT-Perio for individuals with FESS who had perioperative anxiety, depression symptoms, or post-operative insomnia. The intervention appeared to be useful not only in improving anxiety and insomnia symptoms but also in biological parameters (cortisol) compared to the control group. However, there were no RCTs reported about CCBT treating surgical patients with diverse comorbidities of mental disorders. The present study provides preliminary data to assess the potential efficacy and mechanism of mobile technology-based CCBT interventions in clinical surgery applications.

In the present study, table 2 demonstrates how the outcomes between and within groups differed significantly in STAI-S and AIS, indicating that patients in the CCBT group experienced alleviated anxiety and insomnia symptoms at T2/T3 and T4, compared to UC group. Figure 3 illustrated that percentage of patients who were moderate and severe anxious at T2 and post-operative insomnia at T4 in the CCBT group were lower than UC group. The above results showed that the CPCBT-Perio program can help patients scheduled FESS to relieve preoperative anxiety and post-operative insomnia. The results were consistent with the results of recent meta-analyses $[45,46]$, and CCBT was found to improve self-reported psychological outcomes (anxiety and depression) and physical outcomes (pain and insomnia) of patients with psychiatric and somatic disorders. Tellez et al. found that a newly developed computerized cognitive-behavioral therapy was efficacious in reducing anxiety and fear/avoidance of dental surgical procedures at a 1-month follow-up.

Postoperative insomnia may correlate with temporary post-operation nasal tamponade, pain, and bleeding [47]. Moreover, anesthesia-induced disruptions in circadian rhythms may represent a contributing factor to postoperative insomnia [38]. Furthermore, sleep problems can interact with other biological systems; a systematic review proposed that the HPA axis may be a crucial linking sleep in stress-related pathology [48]. Sleep problems can also affect the secretion of cortisol, and adults with sleep problems and psychological disorders exhibit higher levels of cortisol. Increased cortisol and an imbalance of the HPA axis can contribute to the development of anxiety and depression [48]. On the contrary, anxiety and depression can also worsen insomnia. Yang et al. put forward that not only physiological factors but also the psychological factors of emotional distress affect postoperative sleep quality; anxiety and depression was a significant predictor of sleep quality [9]. However, no significant effects were observed for depression symptoms between the two groups, which may be due to surgery- 
related stress being experienced on a short-term timescale, while depression may exhibit a more extended time course. For example, Yang et al. found the majority of for coronary artery bypass graft patients had normal levels of anxiety at one week (69.0\%) and one month (88.5\%) after surgery; however, some level of depression was seen at one week (78.1\%) and one month (59.7\%) [9]. Depression may be less transient and more enduring than state anxiety affected by stress events, which examine moment-to-moment state affective constructs. The intervention's focus on these targets may explain its specific effects on these outcomes, with lesser effects on long-term [44], mainly since scores for depression (PHQ-9) were relatively low at baseline and showed minimal changes.

We further found that increases in cortisol level of the UC group were significantly more higher at T2 compared to T1; increases in cortisol level at T2 were significantly UC group compared to CCBT group. This result showed that CPCBT-Perio could decrease cortisol levels in the CCBT group and supported previous views that cognitive interventions can significantly reduce cortisol and ACTH [41]. The cortisol level is currently the most accurate among several common indicators to assess patients' level of anxiety since it is not affected by measurement conditions [49]. We found the mean serum cortisol level to be lower in the CCBT group than in the UC group. Asghari et al. reported similar findings that cortisol levels were significantly reduced because of preoperative nursing visits for patients operated on ear, nose, and throat problems compared with the control group [50]. For instance, Usha et al. found that Rajyoga meditation as a form of mind-body intervention can positively affect modulating cortisol levels in patients undergoing major cardiac surgery. Acute stress, whether environmental or psychological, results in the release of ACTH from the anterior pituitary gland and an elevation in circulating cortisol [51]. Cortisol homeostasis is essential for cognitive and affective functions that depend on cortisol sensitive brain regions, including the hippocampus and PFC. However, in the present study, the change of ACTH between the two groups was not visible. It may be related to small sample size and short intervention time. In the study, $60 \%$ of participants accepted assay of Cortisol and ACTH because patients declined a traumatic test. The two sessions of the intervention were administered before surgery to decrease preoperative anxiety. The effect of CCBT on hormonal levels may not have been as noticeable as they would have been administered all interventions. McRee and Pasvogel believed that no significant hormonal change might have been caused by the short time interval between the intervention and measurement of cortisol levels [52]. Future studies should explore whether CCBT may cause further changes in physiology following additional repetitions of CCBT interventions.

Vital signs are the physiological indicators that change in stressful situations such as hospitalization or waiting for surgery. The result about present study showed CCBT had an obvious effect on BP and HR change during preoperative period. With the completion of the operation, CCBT had no significant impact on vital signs during post-operative period. Numerous studies and meta-analyses have linked BP responses to mental stress to poor health outcomes [3]. Waiting for operation as a acute stressor, stabilizing preoperative vital signs contributes to good health outcomes. Gu et al. in their study relieved anxiety and stabilized BP and HR by dexmedetomidine combined with parecoxib before anesthesia induction. In present study, CCBT had an similar effect on stabilizing preoperative vital signs as sedation 
and CCBT had a limited impact on vital signs during the postoperative recovery period which can help conduct clinical apply and more research work.

Making the patient safe and comfortable during the whole perioperative period is our medical aim. Satisfaction scores were universally high in CCBT group. There were significant differences between the two groups. However, differences in Disease Care item was no observed, which may be correlated to accept standard nursing procedures. The patients participating in the CCBT group indicated that they received more services, especially psychological services, and more satisfactory medical experience than the past, which makes the relationship between medical staff and patients harmonious, which reduces the incidence of violence [53].

The study had several limitations. Firstly, the intervention was conducted at a single site, with a relatively small sample size, which may have been insufficient and subject to type-ll errors during analysis.

Secondly, the CPCBT-Perio program was only used on patients undergoing FESS, and its efficacy has not been tested in other types of surgery. Thirdly, this study did not explore other potential predictors of patient anxiety, such as income level, cultural structure, surgical categories, disease stage, quality of life, or social support. For instance, CCBT may be less effective in a less-educated population, as internet access and computer experience are less widespread in such populations [44]. Fourthly, our study did not assess the long-term effects in patients, which can be rectified in a future study that continues to follow up on these patients and collect additional data to determine the long-term effects of CPCBT-Perio program. Finally, Further classifications of relevant patient populations will enable CPCBT-Perio software to become more personalized and targeted. As the CPCBT-Perio program is constantly upgraded, future studies will be able to expand the target population and more widely research the cost-effectiveness of these interventions.

\section{Conclusion}

In conclusion, we found that the optimized CPCBT-Perio program may be a suitable and effective adjunctive treatment for patients undergoing FESS. CPCBT-Perio program relieved preoperative anxiety and postoperative insomnia and improved patient satisfaction regarding medical and nursing care. Our findings suggested that CCBT may improve existing nursing practices and promote the development of multidisciplinary treatment.

\section{Abbreviations}

Functional Endoscopic Sinus Surgery (FESS)

Chronic Rhino Sinusitis(CRS)

Cognitive behavior therapy (CBT)

Computer-assisted Cognitive-behavior therapy(CCBT) 
Computer-assisted Psychosomatic Cognitive Behavioral Therapy during perioperative period (CPCBTPerio)

Usual-Care (UC)

Enhanced Recovery After Surgery (ERAS)

State-Trait Anxiety Inventory-State Anxiety (STAI-S)

State-Trait Anxiety Inventory-Trait Anxiety (STAI-T)

Patients Health Questionnaire Depression Scale-9 item (PHQ-9)

Athens Insomnia Scale (AIS)

Cortisol (Cor)

Adrenocorticotropic Hormone (ACTH)

Hypothalamic-Pituitary-Adrenal (HPA)

chemiluminescence assay (CLIA)

Systolic Blood Pressure (SBP)

Diastolic Blood Pressure (DBP)

Heart Rate (HR)

\section{Declarations}

\section{Data statement}

The dataset used and analyzed during the current study is available from the corresponding author on reasonable request.

\section{Acknowledgments}

We would like to thank all participants who made this study possible, and all staffs who participated sufficiently in the work. For the data analysis, we thank Shanxi Hengtai - yifeng Science and Technology Development CO., LTD.

\section{Author contributions}

YY drafted the manuscript. YY and HBZ collected, managed and analyzed data. .YY and YX designed this study. YLL, YX, BQW and $Y Y$ revised the manuscript. BQW and $Y X$ contributed conception and supervision 
the study. All authors approved the final version of the manuscript and agreed to be accountable for all aspects of the work to ensure that questions related to the accuracy or integrity of any part of the work appropriately resolved. Neither the authors' institutions nor the funder had any authority over the trial activities or the preparation of the manuscript.

\section{Availability of data and materials}

The dataset used and analyzed during the current study is available from the corresponding author on reasonable request.

\section{Funding}

This study was supported by the 136 Medical Rejuvenation Project of Shanxi Province, the health research project of Health Commission of Shanxi Province (2018046) and the soft science research plan project of the Shanxi Science and Technology Department (2018041029-6). The funding had no further role in the study design; in the collection, analysis, and interpretation of data; in the writing of the report; and the decision to submit the paper for publication.

\section{Competing interests}

The authors declare that they have no competing interests.

\section{Patient consent}

Obtained.

\section{References}

1. Shi, J.B., et al., Epidemiology of chronic rhinosinusitis: results from a cross-sectional survey in seven Chinese cities. Allergy, 2015. 70: p. 533-539.

2. Nose Section, E.B., Chinese Journal of Otolaryngology and Head and Neck Surgery and O.H.a.N.S.B. Nose Science Section, Chinese Medical Association Chinese guidelines for diagnosis and treatment of chronic rhinosinusitis (2018). Chinese Journal of Otorhinolaryngology Head and Neck Surgery, 2019. 54(2): p. 81-100.

3. O'Connor, D., J. Thayer, and K. Vedhara, Stress and Health: A Review of Psychobiological Processes. Annu. Rev. Psychol., 2020. 72(4): p. 1 26.

4. Caumo, W., et al., Risk factors for preoperative anxiety in adults\&nbsp. Acta Anaesthesiologica Scandinavica, 2001. 45(3): p. 298-307.

5. $\mathrm{H}$, A., et al., A cross-sectional study on preoperative anxiety in adults. Journal of psychosomatic research, 2018. 111: p. 133-139.

6. Caumo, W., et al., Risk factors for preoperative anxiety in adults. Acta Anaesthesiologica Scandinavica, 2010. 45(3): p. 298-307. 
7. Luo, M., B. Song, and J. Zhu, Sleep Disturbances After General Anesthesia: Current Perspectives. Frontiers in neurology, 2020. 11: p. 629.

8. F, C., et al., Postoperative sleep disruptions: a potential catalyst of acute pain? Sleep medicine reviews, 2014. 18(3): p. 273-82.

9. PL, Y., et al., Sleep Quality and Emotional Correlates in Taiwanese Coronary Artery Bypass Graft Patients 1 Week and 1 Month after Hospital Discharge: A Repeated Descriptive Correlational Study. PloS one, 2015. 10(8): p. e0136431.

10. Song Bijia, C.Y., Zhu Junchao, The effect of general anesthesia on sleep rhythm of patients. Journal of Medical Postgraduates, 2018. 31(11): p. 93-96.

11. Seok, J., et al., Psychological and neuroendocrinological characteristics associated with depressive symptoms in breast cancer patients at the initial cancer diagnosis. General hospital psychiatry, 2010. 32(5): p. 503-508.

12. Leonard, B., HPA and immune axes in stress: involvement of the serotonergic system. Neuroimmunomodulation, 2006. 13: p. 268-276.

13. Bahrami, N., et al., Effects of anxiety reduction training on physiological indices and serum cortisol levels before elective surgery. Iranian journal of nursing midwifery research, 2013. 18(5): p. 416-420.

14. Gu, X., et al., The clinical effect of dexmedetomidine combined with parecoxib sodium on sedation, antianxiety and prevention of intubation stress in patients undergoing functional endoscopic sinus surgery: a randomised controlled trial. BMC anesthesiology, 2020. 20(1): p. 166.

15. Beck, A., Thinking and Depression: Il. Theory and Therapy. Archives of General Psychiatry, 1964. 10(6): p. 561-571.

16. Dao, T.K., et al., Randomized controlled trial of brief cognitive behavioral intervention for depression and anxiety symptoms preoperatively in patients undergoing coronary artery bypass graft surgery. The Journal of Thoracic and Cardiovascular Surgery, 2011. 142(3): p. 109-115.

17. JA, C., A. DK, and L. SE, A roadmap to computer-based psychotherapy in the United States. Harvard review of psychiatry, 2010. 18(2): p. 80-95.

18. S, v.d.B., et al., Computerized cognitive-behaviour therapy for anxiety and depression: a practical solution to the shortage of trained therapists. Journal of psychiatric and mental health nursing, 2004. 11(5): p. 508-13.

19. JH, W., et al., Computer-Assisted Cognitive-Behavior Therapy in Medical Care Settings. Current psychiatry reports, 2018. 20(10): p. 92.

20. G, A., et al., Computer therapy for the anxiety and depression disorders is effective, acceptable and practical health care: An updated meta-analysis. Journal of anxiety disorders, 2018. 55(undefined): p. 70-78.

21. A, R., et al., Computerized Cognitive Behavioral Therapy for Treatment of Depression in Multiple Sclerosis: A Narrative Review of Current Findings and Future Directions. International journal of MS care, 2019. 21(3): p. 113-123. 
22. M, B., et al., Internet-Delivered Exposure-Based Cognitive-Behavioral Therapy for Adolescents With Functional Abdominal Pain or Functional Dyspepsia: A Feasibility Study. Behavior therapy, 2019. 50(1): p. 177-188.

23. CR, J., et al., Feasibility of implementing mobile technology-delivered mental health treatment in routine adult sickle cell disease care. Translational behavioral medicine, 2020. 10(1): p. 58-67.

24. SK, S., et al., Computerized Cognitive Behavioral Therapy to Treat Emotional Distress After Stroke: A Feasibility Randomized Controlled Trial. JMIR mental health, 2017. 4(2): p. e16.

25. M, T., et al., Computerized Tool to Manage Dental Anxiety: A Randomized Clinical Trial. Journal of dental research, 2015. 94: p. 174S-80S.

26. D, M., et al., CONSORT 2010 explanation and elaboration: updated guidelines for reporting parallel group randomised trials. BMJ, 2010. 340: p. c869.

27. Kroenke, K., R.L. Spitzer, and J.B.W. Williams, The PHQ-9 : Validity of a Brief Depression Severity Measure. Journal of General Internal Medicine, 2001. 16(9): p. 606-13.

28. Chen Bing, C.Y., Dong Hailong etc., Chinese consensus and clinical guidelines for Enhanced Recovery After Surgery (2018 editon). Chinese Journal of Practical Surgery, 2018. v.38(01): p. 1-20.

29. Spielberger, C., et al., Manual for the State-Trait Anxiety Inventory (Form Y1 - Y2). Vol. IV. 1983, Redwood City,: CA: Consulting Psychologists Press.

30. I, T. and R. JS, Assessment and Management of Preoperative Anxiety. Journal of voice : official journal of the Voice Foundation, 2019. 33(5): p. 691-696.

31. Julian, L., Measures of anxiety: State-Trait Anxiety Inventory (STAI), Beck Anxiety Inventory (BAI), and Hospital Anxiety and Depression Scale-Anxiety (HADS-A). Arthritis care research in nursing \& health, 2011: p. S467-72.

32. Shek, D.T.L., Reliability and factorial structure of the Chinese version of the State-Trait Anxiety Inventory. 1988. 10(4): p. 303-317.

33. NH, B., et al., Preoperative anxiety: detection and contributing factors. Canadian journal of anaesthesia = Journal canadien d'anesthesie, 1990. 37: p. 444-7.

34. Pan X, et al., Related risk factors of moderate and severe preoperative anxiety. Journal of Clinical Anesthesiology, 2018. 5(34): p. 425-428.

35. Ran, C., et al., Evaluation of the reliability and validity of PHQ-9 in general hospital inpatients. Sichuan Mental Health, 2017. 30(2): p. 149-53.

36. M, S. and S. U, Longitudinal changes in depression screening results in cardiac surgery patients. Journal of thoracic disease, 2019. 11(3): p. 920-926.

37. Soldatos, C.R., D.G. Dikeos, and T.J. Paparrigopoulos, Athens Insomnia Scale: Validation of an instrument based on ICD-10 criteria. Journal of Psychosomatic Research, 2000. 48(6): p. 555-60.

38. G, D., et al., Desynchronization of daily rest-activity rhythm in the days following light propofol anesthesia for colonoscopy. Clinical pharmacology and therapeutics, 2009. 85(1): p. 51-5. 
39. Soldatos, C.R., D.G. Dikeos, and T.J. Paparrigopoulos, The diagnostic validity of the Athens Insomnia Scale. Journal of Psychosomatic Research, 2003. 55(3): p. 260-67.

40. Zarei, B., et al., The effect of multimedia-based nursing visit on preoperative anxiety and vital signs in patients undergoing lumbar disc herniation surgery: A randomised clinical trial. 2018: p. 7-15.

41. JL, A., et al., Effects of perceived control and cognitive coping on endocrine stress responses to pharmacological activation. Biological psychiatry, 2008. 64(8): p. 701-7.

42. Jingwei, Z., Z. Wei, and W. Zhun, A survey of pre-anesthesia anxiety and analysis of risk factors. Chinese Journal of Anesthesiology, 2019. 39(6): p. 673-675.

43. C, R., V. E, and R. G, Effectiveness of an informative video on reducing anxiety levels in patients undergoing elective coronarography: an RCT. European journal of cardiovascular nursing, 2009. 8(1): p. 57-61.

44. Nikrahan, G., et al., Randomized controlled trial of a well-being intervention in cardiac patients. General hospital psychiatry, 2019. 61: p. 116-124.

45. Yang, L., et al., Application and research progress of internet-based cognitive behavioral therapy for insomnia disorder. Journal of Southern Medical University, 2020. 40(1): p. 142-146.

46. Mehta, S., V. Peynenburg, and H. Hadjistavropoulos, Internet-delivered cognitive behaviour therapy for chronic health conditions: a systematic review and meta-analysis. Journal of behavioral medicine, 2019. 42(2): p. 169-187.

47. Li, L., et al., Insomnia after functional endoscopic sinus surgery:a case report. Chinese journal of otorhinolaryngology head, 2015. 50(2): p. 158-9.

48. JH, v.D. and M. CR, The influence of sleep on human hypothalamic-pituitary-adrenal (HPA) axis reactivity: A systematic review. Sleep medicine reviews, 2018. 39: p. 187-194.

49. Hek, K., et al., Anxiety disorders and salivary cortisol levels in older adults: a population-based study. Psychoneuroendocrinology, 2013. 38(2): p. 300-305.

50. K, A., et al., Effect of Preoperative Nursing Visit on Serum Cortisol Levels in PatientsWaiting for Operation:A Randomized Study with Control Group. Med J Tabriz Univ Med Sci 2009. 30: p. 13-16.

51. BE, L., The HPA and immune axes in stress: the involvement of the serotonergic system. European psychiatry : the journal of the Association of European Psychiatrists, 2005. 20: p. S302-6.

52. LD, M., N. S, and P. A, Using massage and music therapy to improve postoperative outcomes. AORN journal, 2003. 78(3): p. 433-447.

53. Y, M., et al., Development of two psychological experience questionnaires for screening violencerelated mental health disorders of non-psychiatric inpatients. Health and quality of life outcomes, 2020. 18(1): p. 151.

\section{Figures}


Dayo Day1 Day2 Day3 Operation Day 1 Day 2 Day 3 Day 4 Day 5

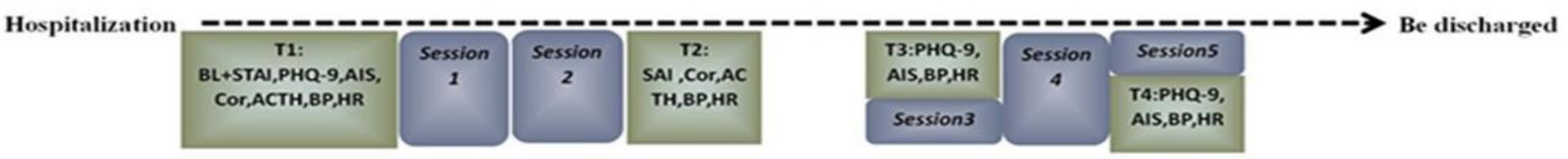

BL: baseline assessment; STAI: State-Trait Anxiety Imventory : S.A: State Anxiety Inventory: PH1-9: Paticnt Health Questionnaire Depression Scale-9: AIS: Athens Insomnia Seale; Cor: Cortisol: ACTH:Adrenocorticotropic Hormone: BP: Blood Pressure: HR: Heart Rate; Session: intervention.

\section{Figure 1}

The schedule of study assessment, and intervention time points

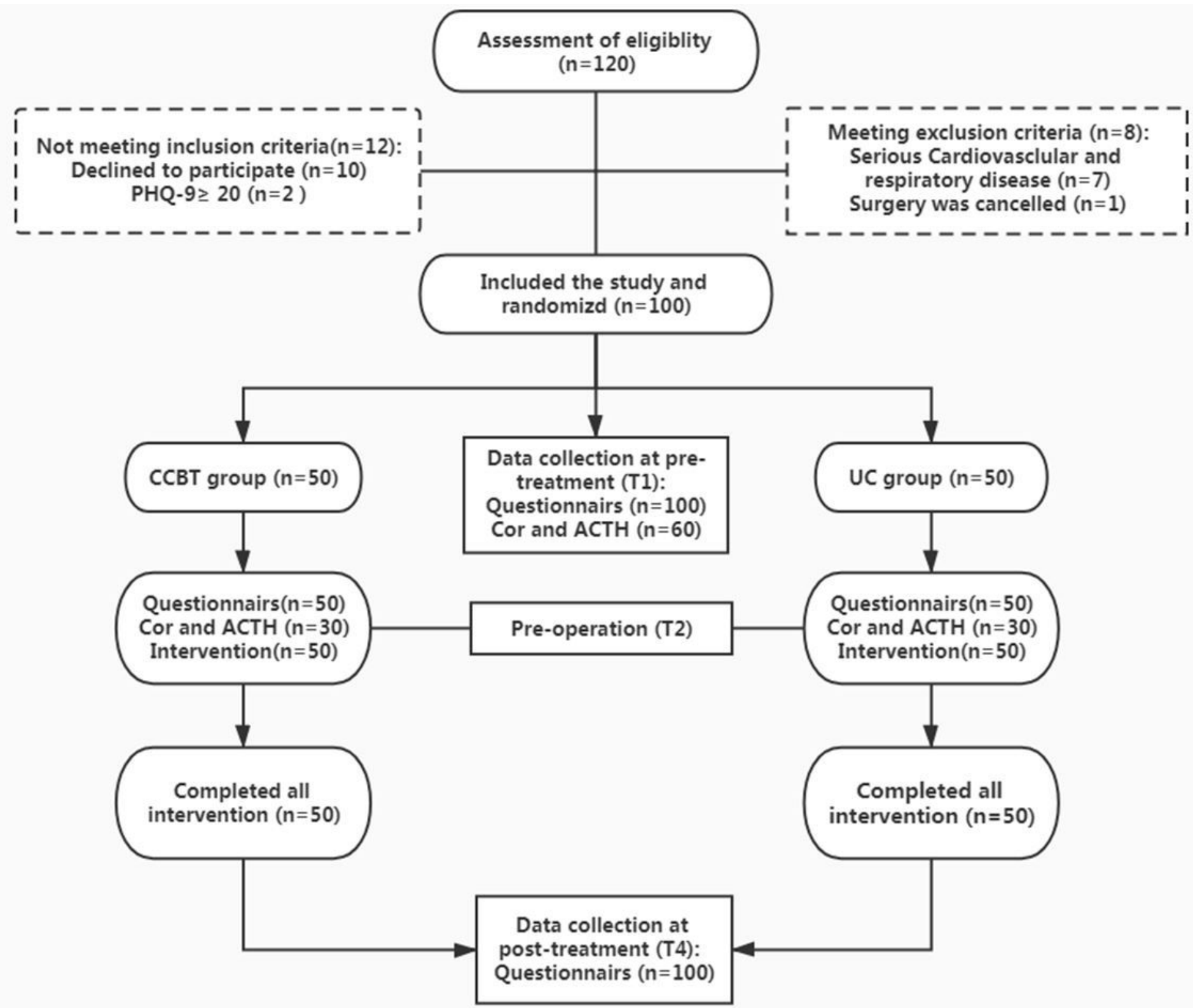

Figure 2

CONSORT flow diagram 
The change trend line chart of score
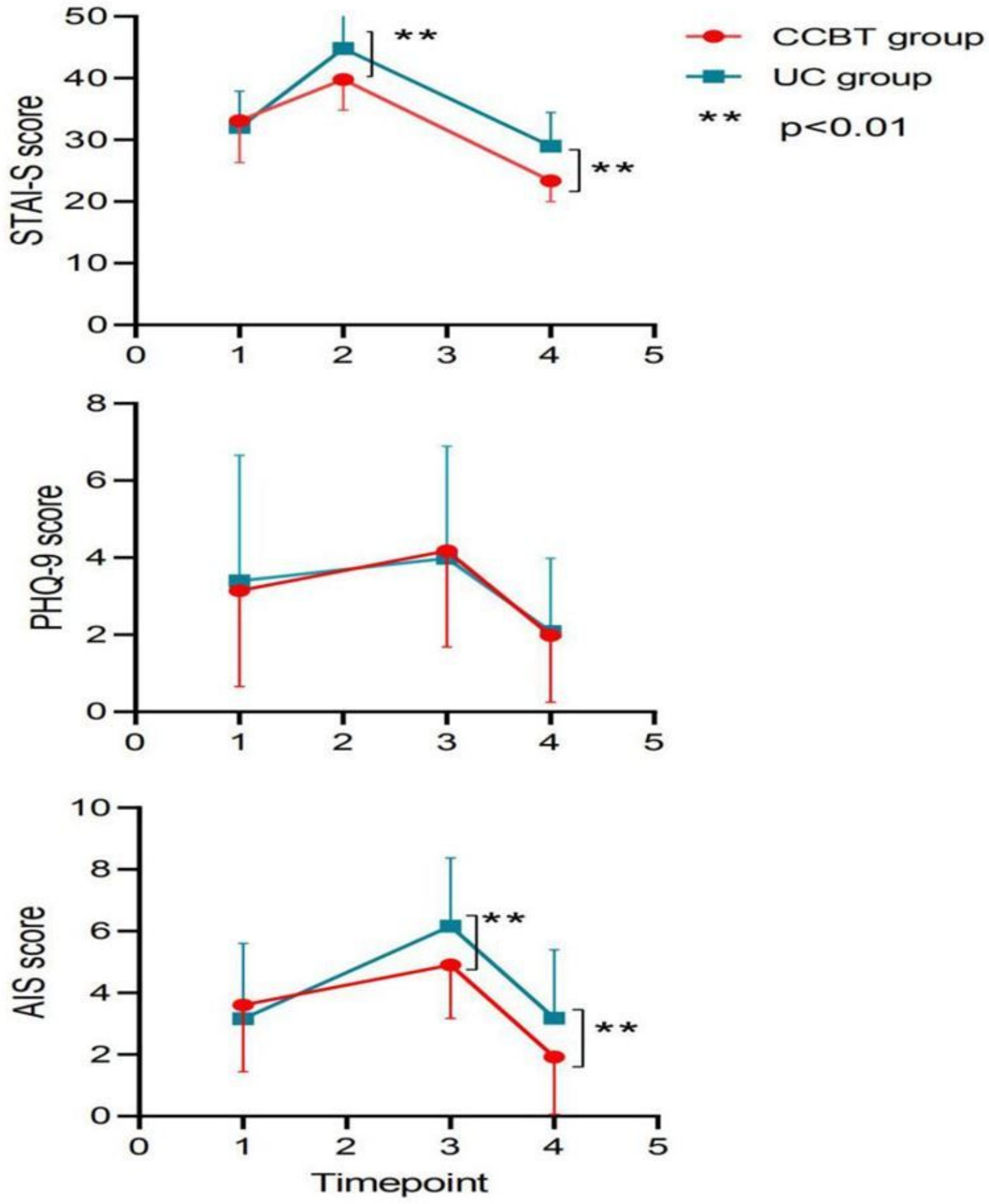

Figure 3

The comparison about change trend of scores between-two groups 
Participants falling above the clinical cut-off score

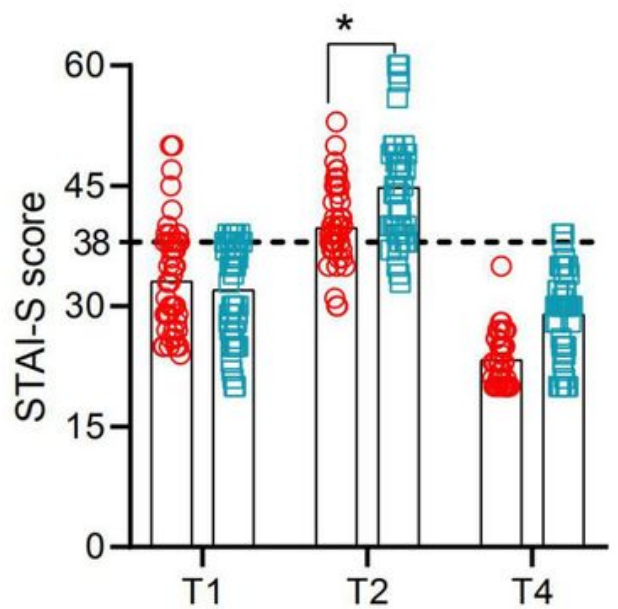

$\circ \quad$ CCBT group
$\square \quad$ UC group
$* \quad p<0.05$
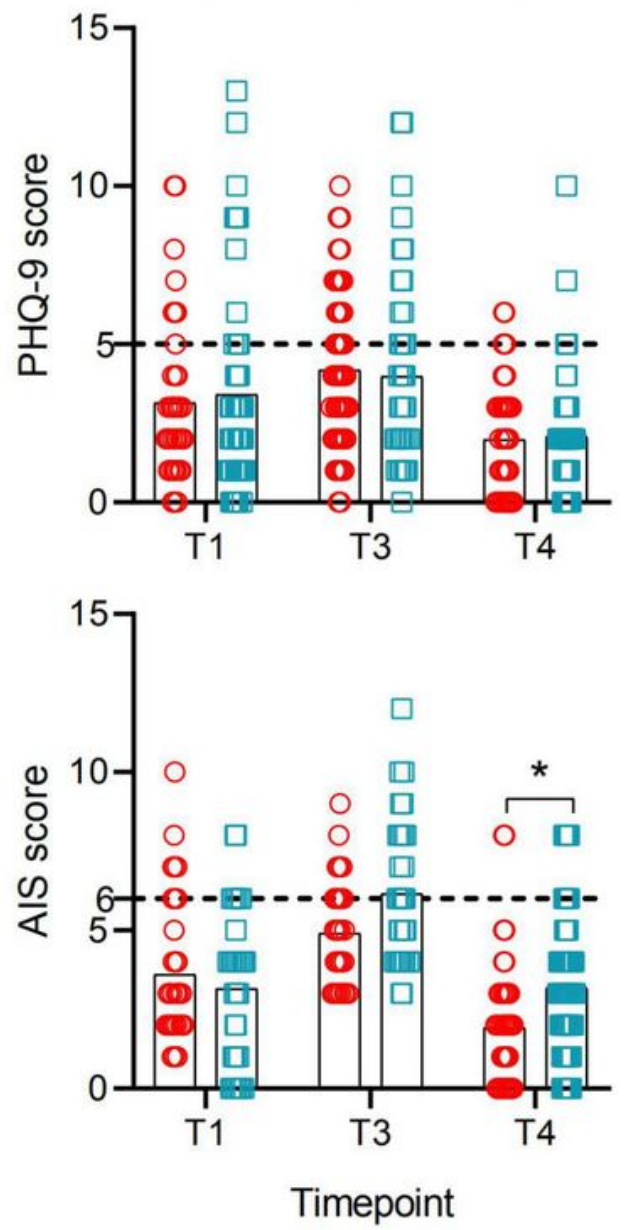

Figure 4

The comparison about participants falling above the clinical cut-off score 
The change trend of systolic blood pressure

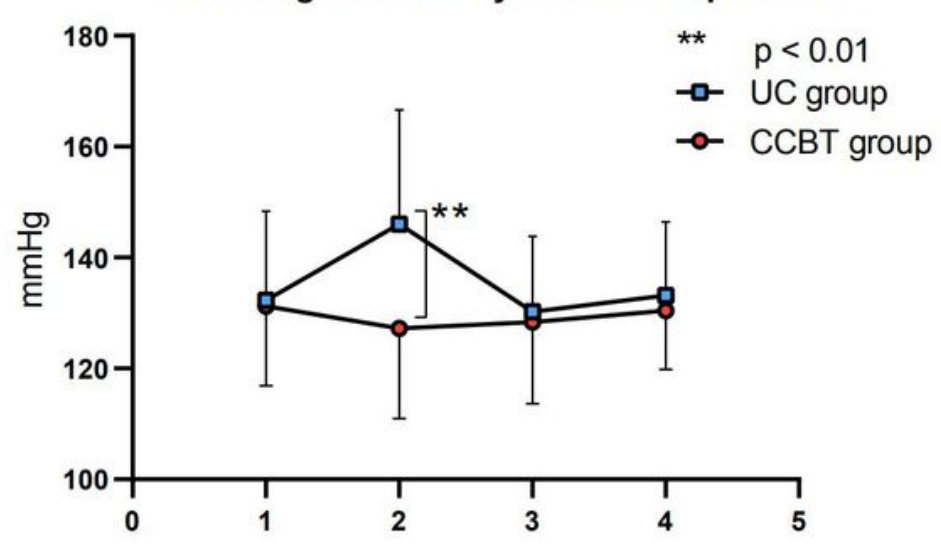

The change trend of diastolic blood pressure

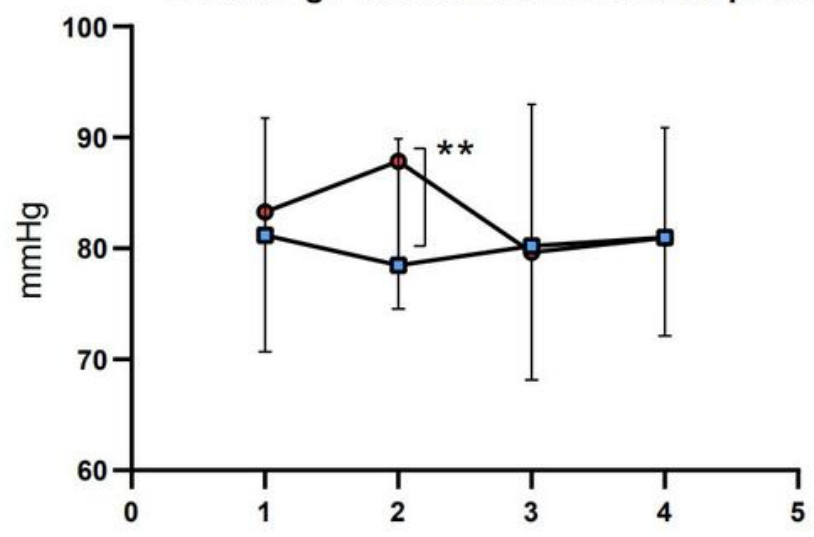

The change trend of heart rate

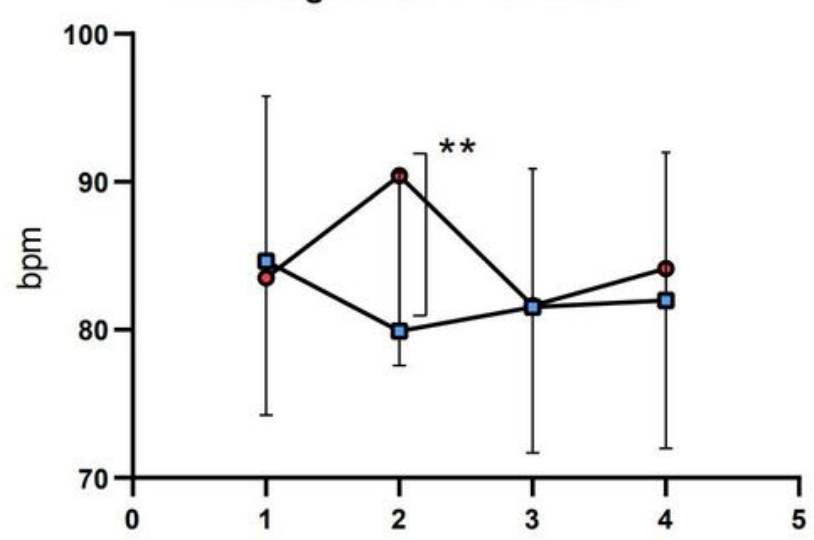

Figure 5

The change trend line chart of $\mathrm{BP}$ and $\mathrm{HR}$

\section{Supplementary Files}

This is a list of supplementary files associated with this preprint. Click to download. 
- supplementtable.docx

Page 25/25 